\title{
Quality control of B-lines analysis in stress Echo 2020
}

Maria Chiara Scali ${ }^{17,40}$, Quirino Ciampi ${ }^{1,2^{*}}$, Eugenio Picano ${ }^{1}$, Eduardo Bossone ${ }^{18}$, Francesco Ferrara ${ }^{18}$, Rodolfo Citro $^{3}$, Paolo Colonna ${ }^{4}$, Marco Fabio Costantino ${ }^{5}$, Lauro Cortigiani ${ }^{6}$, Antonello D'. Andrea ${ }^{7}$, Sergio Severino ${ }^{7}$, Claudio Dodii ${ }^{8}$ Nicola Gaibazzi ${ }^{9}$, Maurizio Galderisi ${ }^{10}$, Andrea Barbieri ${ }^{11}$, Ines Monte ${ }^{12}$, Fabio Mori ${ }^{13}$, Barbara Reisenhofer ${ }^{14}$, Federica Re ${ }^{15}$, Fausto Rigo ${ }^{16}$, Paolo Trambaiolo ${ }^{19}$, Miguel Amor ${ }^{20}$, Jorge Lowenstein ${ }^{21}$, Pablo Martin Merlo ${ }^{21}$, Clarissa Borguezan Daros ${ }^{22}$, José Luis de Castro e Silva Pretto ${ }^{23}$, Marcelo Haertel Miglioranza ${ }^{24}$, Marco A. R. Torres ${ }^{25}$, Clarissa Carmona de Azevedo Bellagamba ${ }^{25}$, Daniel Quesada Chaves $^{26}$, lana Simova ${ }^{27}$, Albert Varga ${ }^{28}$, Jelena Čelutkiené ${ }^{29}$, Jaroslaw D. Kasprzak ${ }^{30}$, Karina Wierzbowska-Drabik ${ }^{30}$, Piotr Lipiec ${ }^{30}$, Paulina Weiner-Mik ${ }^{30}$, Eva Szymczyk ${ }^{30}$, Katarzyna Wdowiak-Okrojek ${ }^{30}$, Ana Djordjevic-Dikic ${ }^{31}$, Milica Dekleva ${ }^{32}$, Ivan Stankovic ${ }^{33}$, Aleksandar N. Neskovic ${ }^{33}$, Angela Zagatina ${ }^{34}$, Giovanni Di Salvo ${ }^{35}$, Julio E. Perez ${ }^{36}$, Ana Cristina Camarozano ${ }^{37}$, Anca Irina Corciu ${ }^{38}$, Alla Boshchenko ${ }^{39}$, Fabio Lattanzi ${ }^{40}$, Carlos Cotrim ${ }^{41}$, Paula Fazendas ${ }^{42}$, Maciej Haberka ${ }^{43}$, Bozena Sobkowic ${ }^{44}$, Wojciech Kosmala ${ }^{45}$, Tomasz Witkowski ${ }^{45}$, Piotr Gosciniak ${ }^{46}$, Alessandro Salustri ${ }^{47}$, Hugo Rodriguez-Zanella ${ }^{48}$, Luis Ignacio Martin Leal ${ }^{40}$, Alexandra Nikolic ${ }^{49}$, Suzana Gligorova ${ }^{50}$, Madalina-Loredana Urluescu ${ }^{51}$, Maria Fiorino ${ }^{52}$, Giuseppina Novo ${ }^{53}$, Tamara Preradovic-Kovacevic ${ }^{54}$, Miodrag Ostojic ${ }^{49,54}$, Branko Beleslin ${ }^{31}$, Bruno Villari ${ }^{2}$, Michele De Nes ${ }^{1}$, Marco Paterni ${ }^{1}$, Clara Carpeggiani ${ }^{1}$ and on behalf of Stress Echo 2020 study group of the Italian Society of Echocardiography and Cardiovascular Imaging (SIECVI)

\section{Abstract}

Background: The effectiveness trial "Stress echo (SE) 2020" evaluates novel applications of SE in and beyond coronary artery disease. The core protocol also includes 4-site simplified scan of B-lines by lung ultrasound, useful to assess pulmonary congestion.

Purpose: To provide web-based upstream quality control and harmonization of B-lines reading criteria.

Methods: 60 readers (all previously accredited for regional wall motion, 53 B-lines naive) from 52 centers of 16 countries of SE 2020 network read a set of 20 lung ultrasound video-clips selected by the Pisa lab serving as reference standard, after taking an obligatory web-based learning 2-h module (http://se2020.altervista.org). Each test clip was scored for Blines from 0 (black lung, A-lines, no B-lines) to 10 (white lung, coalescing B-lines). The diagnostic gold standard was the concordant assessment of two experienced readers of the Pisa lab. The answer of the reader was considered correct if concordant with reference standard reading \pm 1 (for instance, reference standard reading of 5 B-lines; correct answer 4, 5, or 6). The a priori determined pass threshold was 18/20 ( $\geq 90 \%$ ) with $R$ value (intra-class correlation coefficient) between reference standard and recruiting center) $>0.90$. Inter-observer agreement was assessed with intra-class correlation coefficient statistics.

(Continued on next page)

\footnotetext{
* Correspondence: qciampi@gmail.com

${ }^{1} \mathrm{CNR}$, Institute of Clinical Physiology, Biomedicine Department, Pisa, Italy

${ }^{2}$ Cardiology Division, Fatebenefratelli Hospital, Benevento, Italy

Full list of author information is available at the end of the article
}

(C) The Author(s). 2018 Open Access This article is distributed under the terms of the Creative Commons Attribution 4.0 International License (http://creativecommons.org/licenses/by/4.0/), which permits unrestricted use, distribution, and reproduction in any medium, provided you give appropriate credit to the original author(s) and the source, provide a link to the Creative Commons license, and indicate if changes were made. The Creative Commons Public Domain Dedication waiver (http://creativecommons.org/publicdomain/zero/1.0/) applies to the data made available in this article, unless otherwise stated. 


\begin{abstract}
(Continued from previous page)
Results: All 60 readers were successfully accredited: 26 (43\%) on first, 24 (40\%) on second, and 10 (17\%) on third attempt. The average diagnostic accuracy of the 60 accredited readers was $95 \%$, with R value of 0.95 compared to reference standard reading. The 53 B-lines naive scored similarly to the 7 B-lines expert on first attempt (90 versus 95\%, $p=\mathrm{NS})$. Compared to the step-1 of quality control for regional wall motion abnormalities, the mean reading time per attempt was shorter ( $17 \pm 3$ vs $29 \pm 12 \mathrm{~min}, p<.01$ ), the first attempt success rate was higher ( $43 \mathrm{vs} 28 \%, p<0.01$ ), and the drop-out of readers smaller (0 vs $28 \%, p<.01)$.

Conclusions: Web-based learning is highly effective for teaching and harmonizing B-lines reading. Echocardiographers without previous experience with B-lines learn quickly.
\end{abstract}

Keywords: Certification, Lung comets, Quality control, Stress echocardiography, Wall motion

\section{Background}

Stress echocardiography (SE) has some advantages over competing imaging techniques, including low cost, portability, radiation-free nature and versatility. Its major limitation is the dependence upon operator's expertise, which may impact on the quality and consistency of diagnostic results $[1,2]$. This limitation is magnified when the technique is used for scientific purposes in a multi-center trial such as Stress Echo 2020 (SE2020) study, designed to provide effectiveness data in 10,000 patients from $>100$ laboratories in a variety of conditions ranging from coronary artery disease to heart failure (with preserved or depressed ejection fraction), hypertrophic cardiomyopathy, repaired congenital heart disease, valvular heart disease and extreme physiology [3]. To achieve harmonization, one possible approach is the use of the core lab which analyses centrally images sent from all recruiting sites. This approach is typically the preferred choice in a clinical trial and minimizes the sources of measurement variability $[4,5]$. The core lab option was discarded in SE 2020 for two reasons. First, it was too costly and logistically demanding. Second, it would provide efficacy data under ideal conditions, but our aim was to obtain effectiveness data realistically generated when the technique is deployed in the clinical arena, populated by real patients, real doctors and real problems [6]. A feasible approach to ensure consistency in data acquisition and interpretation in this challenging setting is to develop an upstream reading quality control for prospective centers willing to enter the study $[7,8]$. In SE2020, this approach has already been implemented for regional wall motion abnormalities (RWMA), which remains the diagnostic cornerstone of SE [9]. However, a separate quality control needs to be performed for other aspects of contemporary SE practice, such as B-lines obtained with lung ultrasound (LUS) [10]. Also known as ultrasound lung comets, B-lines are a sign of accumulation of extra-vascular lung water [11] and can acutely increase during stress [12-14]. Their presence and/ or increase during stress places the patient in a higher risk subset for any level of RWMA [13] and indicates that dyspnea is linked to acute backward heart failure [15]. B-lines assessment must be properly standardized and quality-controlled prior to dissemination and use for clinical and scientific purposes. The present report was part of the larger SE2020 study and focuses on the educational aspects of LUS-SE, describing the results of the upstream quality control and harmonization of B-lines reading criteria across 52 SE2020 centers.

\section{Methods}

The Pisa lab coordinated the quality control assessment for B-lines of all investigators who expressed their intention to participate in the study (Fig. 1). The coordinating center was in the National Research Council, Institute of Clinical Physiology in Pisa, Italy. The candidate centers included 52 centers (each with at least one certified reader) from 16 countries (Argentina, Brazil, Bulgaria, Costa Rica, Hungary, Italy, Lithuania, Mexico, Poland, Portugal, Romania, Qatar, Russia, Serbia, UK, USA). The selection criterion was that all readers had already passed the quality control for RWMA reading (step 1 in the "Road to SE 2020"). The B-lines reading was the step 2 in the "Road to SE 2020". The complete list of participants in the SE2020 consortium (as per January 20th, 2018) is reported in the Appendix. The study protocol was reviewed and approved by the institutional ethics committee as a part of the SE 2020 study (1487-CE Lazio-1, July 20, 2016). The study was funded with institutional funding of the Italian National Research Council and with travel grants of the Italian Society of Echocardiography and Cardiovascular Imaging with dedicated sessions during national meetings. No fort from industry was asked for or received.

An obligatory web-based educational platform was developed to facilitate the training process. Participating cardiologists were invited by email to join the platform, which was protected by user-specific passwords. The platform includes files and videos with detailed instructions on how to start the training and allows downloading and uploading of external files. The sequence of the certification process and web-based learning has already been 


\section{The Road to Stress Echo 2020}

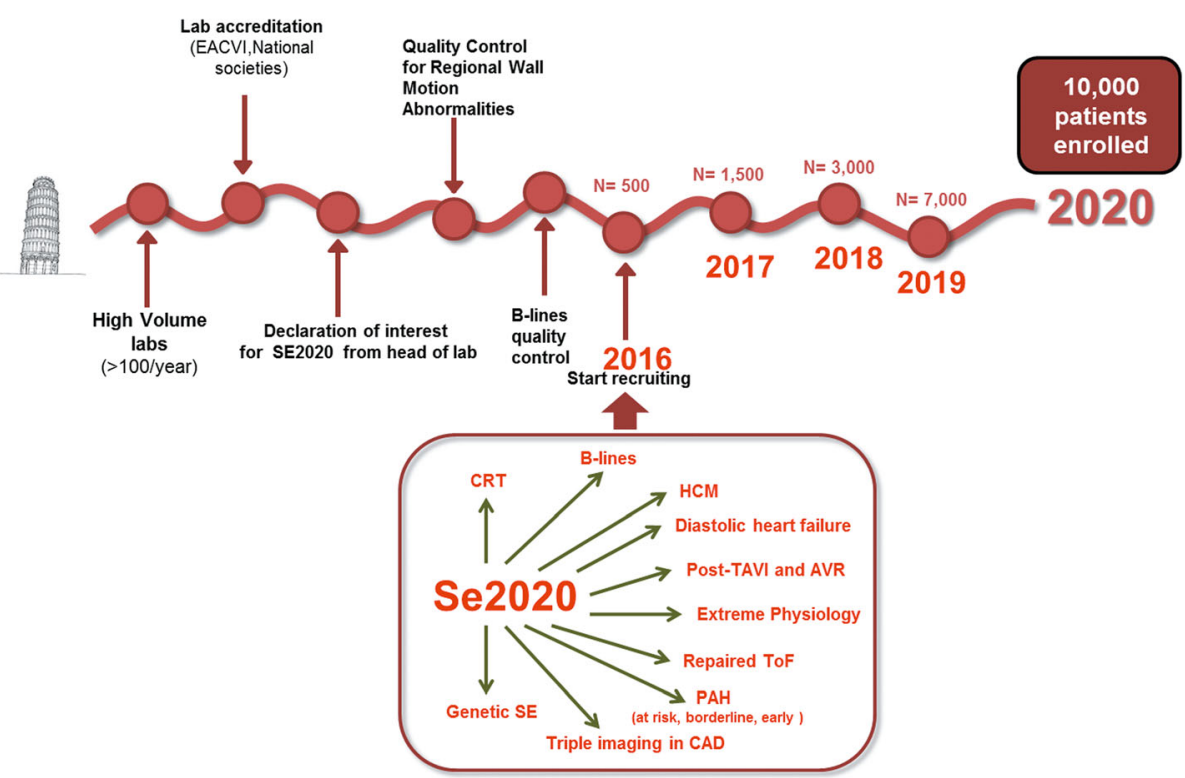

Fig. 1 The road to accreditation for the aspiring recruiting centers. After the first essential step of RWMA, the reader completes the second step (B-lines) and starts enrolling with dual imaging (RWMA and B-lines)

detailed and follows the same template used for RWMA [9]. We decided to have this platform mandatory and not optional as in the step-1 for RWMA, since in case of B-lines the technique is relatively young and recent advances in acquisition (with 4-site scan mode) and reporting were adopted in the SE2020 platform [16].

\section{Study population of readers}

Sixty readers from 52 different centers initially asked to enter the SE2020 study, had passed the RWMA test for quality control and therefore were allowed to enter the step-2 of SE2020.

All participants were clinical cardiologists and expert echocardiographers with ongoing high volume $(>100$ tests per year) SE activity and the years of experience in SE ranged from 5 to 31 years (mean value 18 years). All were certified by national and/or international societies .

\section{Lung ultrasound acquisition}

To acquire lung ultrasound (LUS) images adopted for quality control test, we used commercially available ultrasound machines (IE 33, Philips, Medical Systems, Andover, Massachusetts, USA with a $2.5-3.5 \mathrm{MHz}$ phased-array sector scan probe; Vivid E9, GE Healthcare, USA, manufactured in Horten, Norway, equipped or standard M5S transducer with second harmonic technology; Mylab Eight platform Esaote, Genova, Italy). The depth was adjusted according to the body habitus of the patient, with thin patients requiring less depth and obese patients needing greater depth to visualize the pleural line. A B-line was defined with 4 constant criteria: vertical, laser-like, hyperechoic reverberation; arises from the pleural line extending to the bottom of the screen without fading; moves synchronously with lung sliding; and erases the A-lines, which are a part of the normal lung pattern as a horizontal, multiple reverberation artefact, equidistant from one another below the pleura, at exact multiples of the transducer-pleural line distance [17]. Detailed description of the scanning procedure and scanning sites is also available in a 2-min movie from our laboratory on YouTube (The incredible ULCs - ultrasound lung comets. Available at http://www.youtube.com/ watch?v=7y_hUFBHStM. Accessed: July 10, 2018). LUS scanning was performed with the cardiac probe in the supine position at rest and soon after stress (with the patient again resuming the supine position). The 4-site simplified scan of the lung was used [16]. We analyzed the anterior and lateral hemithoraces, scanning along the anterior axillary (AA) and midaxillary (MA) lines on the third intercostal space (Fig. 2).

\section{Web-based learning module}

The 2-h web-based training module (http://se2020.alter vista.org) consisted of five sequential learning blocks: aSelected readings of 3 recent review or original articles summarizing the evidences supporting the use of B-lines 


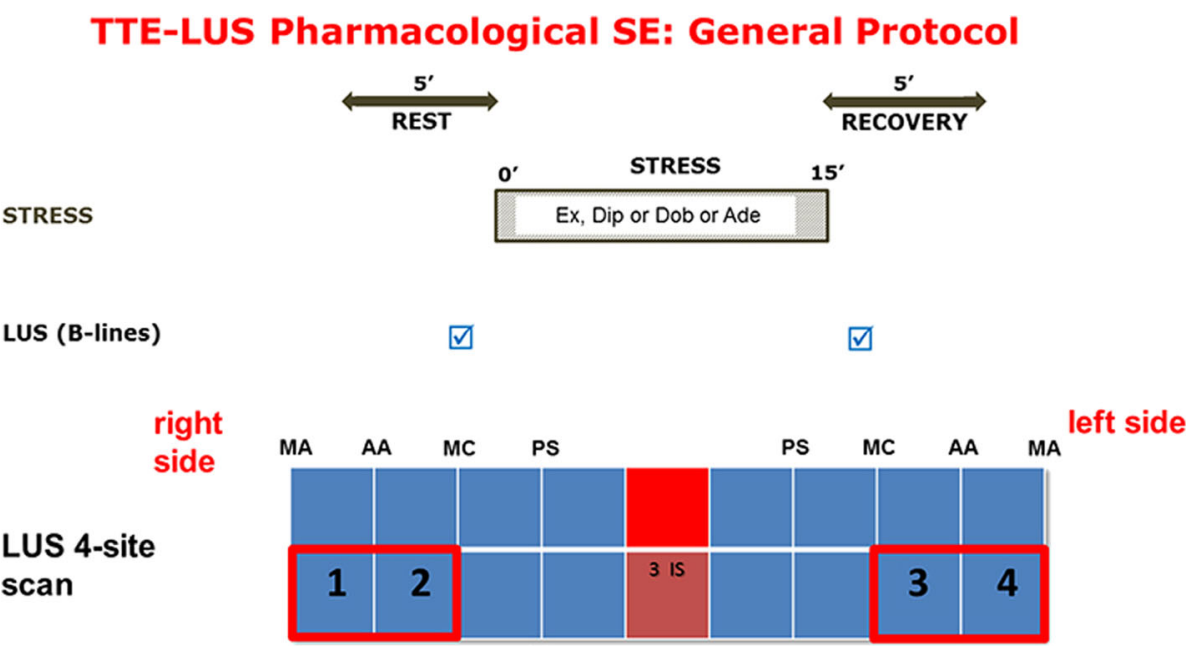

Fig. 2 The Stress-LUS general protocol. LUS for B-lines are assessed at baseline and at the end of stress, after the acquisition for RWMA. The adopted protocol is the 4-site simplified scan

during stress and the adopted scan technique and scoring criteria [8, 10, 13]; b- A power-point file of 25 slides summarizing key points and specific literature supporting the proposed reading policy illustrating tips and tricks highlighting the most frequent problems in B-lines interpretation with special focus on the technicalities of the 4-site simplified scanning technique; c- A theory self-assessment test with five questions with four answers each (only one correct) preliminary to video-clip reading; d- Short $(<15$ s) video-clips of examinations with the same format of official test reading, with $5 \mathrm{~min}$ per reading with countdown clock, and one possible answer (from 0 to 10) for each video-clip (Fig. 3).

An expert trainer (QC or MCS) remained available to all readers for e-mail or phone contact to provide assistance with any issue concerning the training.

At all times there was the possibility of face-to-face discussion (via Skype) to address issues requiring special clarification with the principal investigator. After completing the web-based module the reader could take the test (maximum three attempts). After each attempt, the sequence of videos was mixed.

\section{Reading sessions and pass threshold}

We selected 20 cases of 10 patients (with rest and stress images) in which the presence and number of B-lines was documented by unanimous decision of 2 experienced observers (EP and QC). The privacy of patients during acquisition, storage, and transmission of the SE study was protected. All images were anonymized, and the identity of patients or the study condition (rest or stress) was not disclosed at any time to the readers. Each SE study was structured in a single video-clip of 10$15 \mathrm{~s}$, with either resting or stress images. Each test clip was scored from 0 (black lung, A-lines, no B-lines) to 10 (white lung with coalescing B-lines). The diagnostic gold standard was the reading of Pisa lab. The answer of the reader was considered correct if concordant with reference standard reading \pm 1 (for instance, reference standard reading of 5 B-lines; correct answer 4,5 , or 6 ). The a priori determined pass threshold was $18 / 20$ ( $\geq 90 \%)$ with $\mathrm{R}$ value of intra-class correlation coefficient $>.90$.

The LUS images were selected to represent the garden variety of stress testing modes, responses, results and image quality. They came from six different laboratories (Benevento, Lucca, Pisa, Porto Alegre, Rome, St Petersburg) in three countries (Brasil, Italy, Russian Federation), and showed the full spectrum of responses (from $0, n=7$; to $10, n=1)$. All images were considered readable, with quality ranging from average-to-good $(n=16)$ to excellent $(n=4)$ in the assessment of the reference standard reading. The stress employed was exercise in 17 subjects, high dose accelerated dipyridamole $(0.84 \mathrm{mg} / \mathrm{kg}$ over $6 \mathrm{~min})$ in 2 and dobutamine $(40 \mathrm{mcg} / \mathrm{kg} / \mathrm{min})$ in 1 . The projection selected was the third intercostal space between left mid-axillary and anterior axillary lines in 4; third intercostal space between right mid-axillary and anterior axillary lines in 4; third intercostal space between right anterior-axillary and mid-clavicular lines in 4; third intercostal space between left anterior-axillary and mid-clavicular lines in 8 .

\section{After the pass or fail response}

The response was pass ( $\geq 90 \%$ accuracy) or fail. With pass, the reader received a certificate of accreditation and could start recruiting with a written informed consent signed by each patient and after clearance by the local ethical committee. With fail, the unsuccessful reader could retake the test after 1 month. After the second fail, the reader could undergo training in a recommended center and try again after 1 year. 


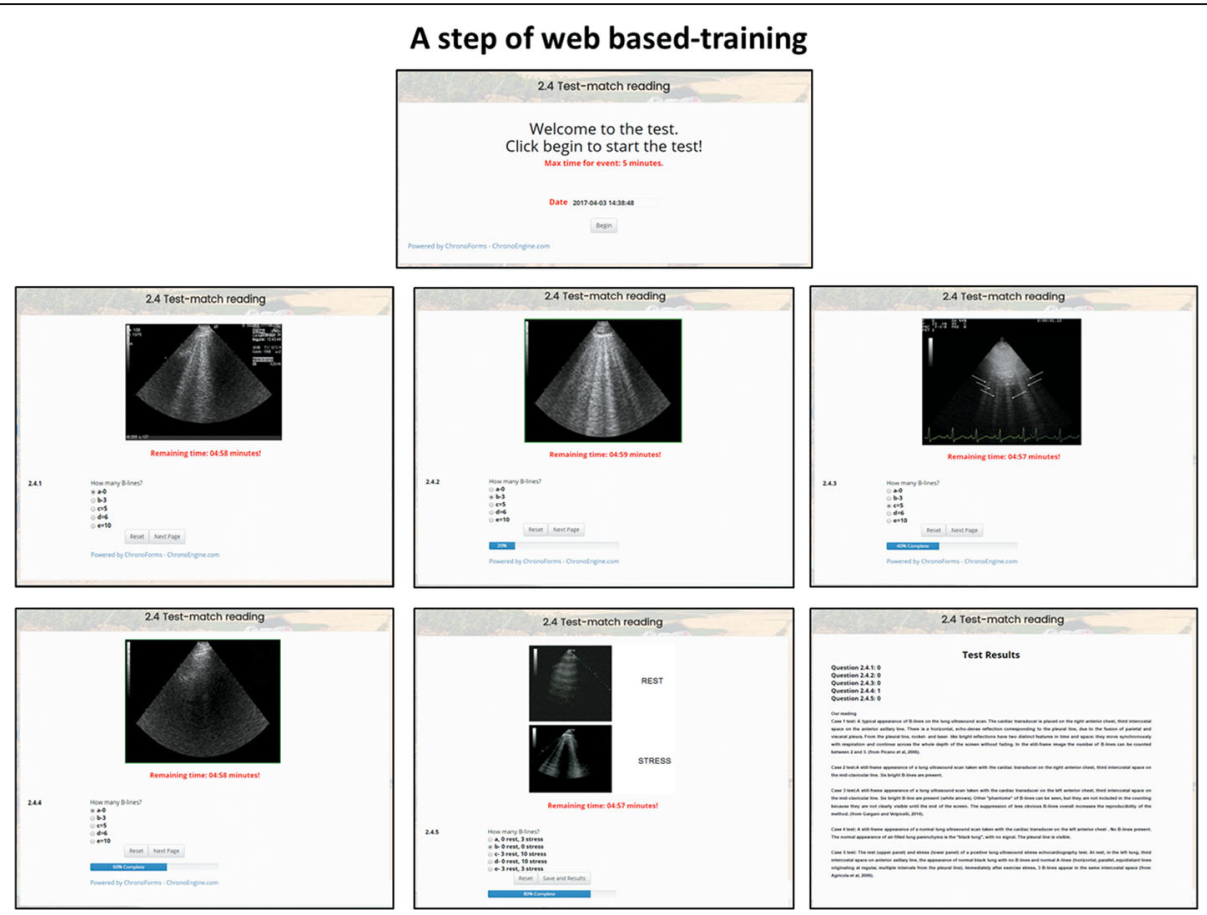

Fig. 3 The screenshot of the test-match step during B-lines quality control. There are 5 still frames (or videos) with B-lines and the trainee has to choose among 5 possible answers, ranging from 0 (left lower panel) to 9 (upper middle panel)

\section{Statistical analysis}

Each reader was evaluated against the gold standard of reference standard reading for assessment of individual accuracy (in \%). The intra-class correlation coefficient was calculated, for each reader, in the whole series of 20 paired measurements made by the peripheral reader and the reference reader. Intra-observer agreement was tested in 20 peripheral readers who volunteered to repeat the measurement session after at least 3 months from the first reading. A $p$ value $<0.05$ was considered significant.

\section{Results}

Of the initial 60 readers who started, 53 were B-lines naive (without previous exposure to B-lines). All 60 readers were successfully accredited (Fig. 4): 26 (43\%) on first, 24 (40\%) on second, and 10 (17\%) on third attempt. The 53 B-lines naive scored similarly to the 7 B-lines expert on first attempt (90 versus $95 \%, p=\mathrm{NS}$ ). Compared to the step-1 of quality control for regional wall motion abnormalities [6], the mean reading time per attempt was shorter $(17 \pm 3$ vs $29 \pm 12 \mathrm{~min}, p<.01)$, the first attempt success rate was higher ( 43 vs $28 \%, p<0.01$ ), and the drop-out of readers smaller ( 0 vs $28 \%, p<.01$ ). The average diagnostic accuracy of the 60 accredited readers was $95 \%$. Considering the final attempt of the 60 readers, the Spearman correlation coefficient between the expert reference reading and the reading of each peripheral reader was very high $(R=0.95, p<.0001)$. In the 20 peripheral readers who repeated the test a second time at least 3 months after accreditation, the Spearman correlation coefficient was also very high $(R=0.97, p<.0001)$.

\section{Discussion}

A user-friendly web-based learning is highly effective for training B-lines also for echocardiographers without previous exposure to B-lines. After a limited learning effort, the accuracy of B-lines reading is comparable between very experienced and freshly trained readers. B-lines with 4-site simplified scan of the lung has a very high success rate in acquisition and analysis. It has been embedded as an integral part of dual imaging SE adopted as the core protocol in SE2020 for all forms of physical and pharmacological stress for all patients, from coronary artery disease to heart failure.

\section{Comparison with previous studies}

The American College of Chest Physicians has defined the knowledge and technical elements required for competence in lung ultrasound [18]. There have been a number of prior lung ultrasound education papers, showing that a limited training of a few hours can improve the capability of execution and interpretation of LUS even in medical students without previous exposure to ultrasound $[19,20]$. In the present study we are dealing with a specific and limited aspect of LUS of special 


\section{Stress Echo 2020 - Quality test}

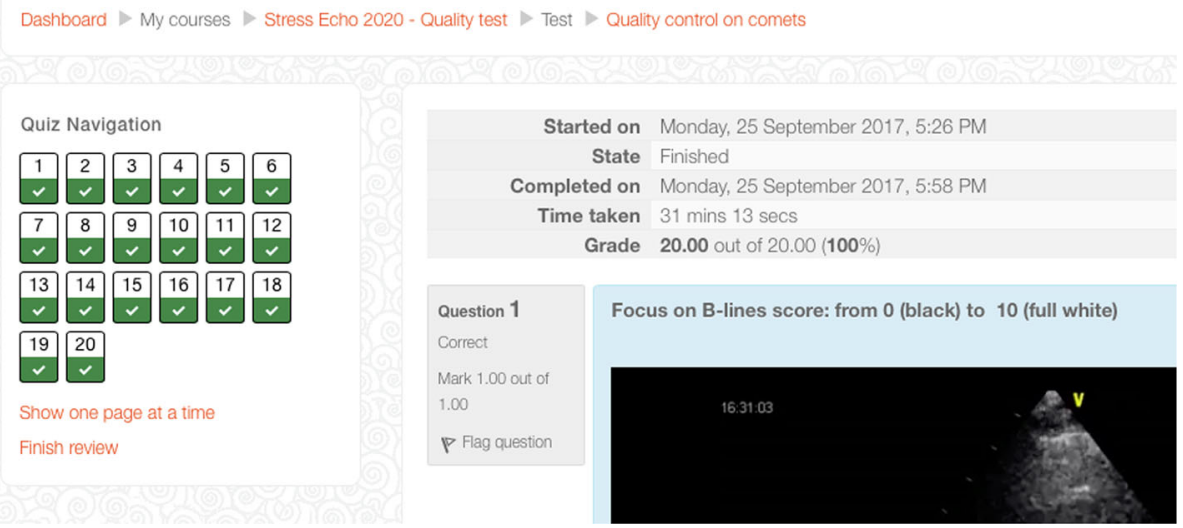

Fig. 4 The test results of a reader passed with full marks (20/20)

interest for cardiologists, i.e. the detection of B-lines. There is a lack of a specific training and certification pathway in cardiology, and as a result training and performance of LUS varies widely among different institutions. An approach similar to the one adopted in the present study was developed in Pisa for centers recruiting in the LUST study [21]. However, this study differs from the previous one under some aspects: first, it was focused on LUS-SE, not on resting LUS; the adopted scan scheme was the simplified 4-site scan, easier to do, to teach and to learn than the previously adopted 28-site scan; and the quality control procedures required some prior reading and slide presentation to facilitate a standardized learning [9].

Our findings are consistent with a large body of literature showing that stable web applications are increasingly used for improving medical image interpretation skills regardless of time and space and without the need for expensive imaging equipment or a patient to scan [22]. With the adopted web-based approach, the educational path is standardized, shared, and - after validation and refinement - prospectively available in open source, and exploitable for scientific purposes and clinical education. The use of enabling technologies makes the accreditation process faster, smoother and cheaper, and coupled with the open-source platform grants an unprecedented opportunity for continuing education, also fostered by endorsement and governance by the scientific society supporting the study.

\section{Study limitations}

We focused on the assessment of B-lines, which is a particularly simple aspect of LUS diagnosis [10, 11]. Similar harmonization and accreditation issues are present for other aspects of SE diagnosis. Separate and parallel training modules are currently under construction within the framework of "SE 2020" to cover the entire spectrum of key aspects of SE diagnosis, from coronary flow velocity reserve to left ventricular volumes and pulmonary hemodynamics [3].

A key aspect in the evaluation of SE results is the adoption of an undisputed diagnostic "gold standard". The lack of a universally acceptable gold standard makes the assessment of reading performance difficult. From the library of images arriving from all the world and stored in our data bank, we selected cases meeting the conditions of unanimous reading of the two most experienced readers from the reference lab. This is a far from perfect gold standard, yet a reasonable, and perhaps the only possible, one.

We restricted our validation phase to participants in the SE2020 study, who had a substantial reading experience and certification in RWMA as a prerequisite. This reader pool may have been especially knowledgeable and motivated, thereby justifying the excellent learning results. However, 53 of them were B-lines naive, and therefore probably the selection criteria of our readers did not affect the generalizability of results.

We adopted a simplified 4-site scan for acquisition of B-lines at rest and during stress. This approach introduces a substantial abbreviation compared to other protocols such as the 28-region scan originally adopted in the Pisa laboratory in the first application of LUS in heart failure patients [23] and also recommended by an international consensus in 2012 [24]. Over the years, simplified 8-zone and 4-zone lung imaging protocols were proposed $[25,26]$, with comparable information between the 2 protocols as shown by Platz et al. [25]. Scali et al. showed that the simplified 4-site scan allows to complete the assessment of B-lines in $20 \mathrm{~s}$ (instead of 
the $3 \mathrm{~min}$ required by the 28 - region scan). There is a linear, close correlation between the 28-site and the 4-site B-lines score [16]. Therefore, there is no significant loss of information when going from 28- to 4-site scan, but a substantial simplification and time saving, vital for SE imaging, when there are so many things to see and so little time available.

\section{Clinical implications}

B-lines are a useful adjunct to mainstream SE based on RWMA [27, 28], but its impact may be limited by the relatively few centers currently using it in their routine SE practice, and the lack of standardization in acquisition, scoring and reporting [29]. After a web-based module and certification, the approach is better harmonized and the accumulation of clinical practice also allows the rapid growth of scientifically unique data. To achieve this goal, simplification is essential, and the 4-site simplified scan is ideal for LUS rest and stress testing.

However, the SE technique does not tolerate improvisation, and an accurate standardization of terminology, standards of execution, and interpretation criteria is required before a center is allowed to enter its experience in the common data bank. Similarly to what has been said for meta-analysis [30], multicenter SE studies are like a bouillabaisse: no matter how much seafood (or recruiting centers) is added, one tainted fish (an unreliable center generating inconsistent reading) will spoil the pot.

\section{Conclusion}

Web-based learning is highly effective for teaching and harmonizing B-lines reading, with an enormous saving of time and resources versus the conventional hands-on approach of teaching and learning ultrasound techniques. Echocardiographers without previous experience with B-lines learn quickly.

\section{Abbreviations \\ CAD: Coronary artery disease; LUS: Lung Ultrasound; RWMA: Regional wall motion abnormalities; SE: Stress echocardiography; TE: Transthoracic echocardiography}

\footnotetext{
Acknowledgments

The study was partially funded with the project Aging of the National Research Council.

On behalf of the Stress Echo 2020 Study Group of the Italian Society of Cardiovascular Echography (as per December 20, 2017). Eugenio Picano', Maria Grazia Andreassi ${ }^{1}$, Clara Carpeggiani ${ }^{1}$, Michele De Nes ${ }^{1}$, Marco Paterni ${ }^{1}$, Lorenza Pratali ${ }^{1}$,Quirino Ciampi ${ }^{2}$, Bruno Villari ${ }^{2}$, Eduardo Bossone ${ }^{3}$, Rodolfo Citro $^{3}$, Francesco Ferrara ${ }^{3}$, Paolo Colonna ${ }^{4}$, Marco Fabio Costantino ${ }^{5}$, Lauro Cortigiani ${ }^{6}$, Antonello D'Andrea ${ }^{7-1}$, Claudio Dodi ${ }^{8}$, Nicola Gaibazzi ${ }^{9}$, Maurizio Galderisi $^{10}$, Andrea Barbieri ${ }^{11}$, Ines Monte ${ }^{12}$, Fabio Mori ${ }^{13}$, lacopo Olivotto ${ }^{13}$, Barbara Reisenhofer ${ }^{14}$, Federica Re $^{15}$, Fausto Rigo ${ }^{16}$, Maria Chiara Scali ${ }^{17,41}$, Sergio Severino ${ }^{7-2}$, Paolo Trambaiolo ${ }^{19}$, Miguel Amor ${ }^{20}$, Jorge Lowenstein ${ }^{21}$, Pablo Martin Merlo ${ }^{21}$, Clarissa Borguezan Daros ${ }^{22}$, José Luis de Castro e Silva Pretto $^{23}$, Marcelo H. Miglioranza ${ }^{24}$, Marco A.R. Torres ${ }^{25}$, Daniel Quesada Chaves $^{26}$, Melissa Rodriguez Israel ${ }^{26}$, lana Simova ${ }^{27}$, Albert Varga ${ }^{28}$, Gergely Agoston ${ }^{28}$, Attila Palinkas ${ }^{28}$, Jelena Čelutkiené ${ }^{29}$, Jaroslaw D. Kasprzak ${ }^{30}$, Karina Wierzbowska-Drabik ${ }^{30}$, Ana Djordjevic-Dikic ${ }^{31}$, Branko Beleslin ${ }^{31}$, '́ilica Dekleva $^{32}$, Aleksandar N. Neskovic ${ }^{33}$, Ivan Stankovic ${ }^{33}$, Angela Zagatina ${ }^{34}$,
}

Giovanni di Salvo ${ }^{35}$, Julio E. Perez ${ }^{36}$, Ana Camarozano ${ }^{37}$, Anca Corciu ${ }^{38}$, Alla Boshcenko ${ }^{39}$, Fabio Lattanzi ${ }^{40}$, Carlos Cotrim ${ }^{41}$, Paula Fazendas ${ }^{42}$, Maciej Haberka ${ }^{43}$, Bozena Sobkowicz ${ }^{44}$, Wojciech Kosmala ${ }^{45}$, Tomasz Witkowski ${ }^{45}$, Piotr Gosciniak ${ }^{46}$, Alessandro Salustri ${ }^{47}$, Hugo Rodriguez Zanella ${ }^{48}$, Alexandra Nikolic ${ }^{49}$, Suzana Gligorova ${ }^{50}$, Madalina-Loredana Urluescu ${ }^{51}$, Maria Fiorino 52 , Giuseppina Novo ${ }^{53}$, Tamara Preradovic-Kovacevic ${ }^{54}$, Miodrag Ostojic ${ }^{33,54}$, Dario Gregori ${ }^{55}$

${ }^{1}$ Institute of Clinical Physiology, National Research Council, Pisa; ${ }^{2}$ Cardiology Division, Fatebenefratelli Hospital, Benevento, Italy; ${ }^{3}$ Cardiology Department and Echocardiography Lab, University Hospital "San Giovanni di Dio e Ruggi d'Aragona", Salerno, Italy; ${ }^{4}$ Cardiology Hospital, Policlinico of Bari, Italy; ${ }^{5}$ Cardiology Department, San Carlo Hospital, Potenza, Italy; ${ }^{6}$ Cardiology Department, San Luca Hospital, Lucca, Italy; ${ }^{7}$ Cardiology Department, Monaldi Hospital, Second University of Naples, Italy; ${ }^{8}$ Casa di Cura Figlie di San Camillo, Cremona; ${ }^{9}$ Cardiology Department, Parma University Hospital, Italy; ${ }^{10}$ Department of Advanced Biomedical Sciences, Federico II University Hospital, Naples, Italy; ${ }^{11}$ Cardiology Department, Modena University Hospital, Modena, Italy; ${ }^{12}$ Cardio-Thorax-Vascular Department, Echocardiography lab, "Policlinico Vittorio Emanuele", Catania University, Italy; ${ }^{13}$ Cardiology Department, Careggi Hospital, Florence, Italy; ${ }^{14}$ Cardiology Division, Pontedera-Volterra Hospital, ASL Toscana 3 Nord-Ovest, Italy; ${ }^{15}$ Cardiology Department, San Camillo-Forlanini Hospital, Roma, Italy; ${ }^{16}$ Cardiology Department, Ospedale dell'Angelo Mestre-Venice, Italy; ${ }^{17}$ Cardiology Department, Nottola Hospital, Siena, and Cardiothoracic Department, University of Pisa, Italy; ${ }^{18}$ Cardiology Department, Ospedale Santa Maria Incoronata dell' Olmo, Cava de' Tirreni, Salerno, Italy; ${ }^{19}$ Department of Cardiology, Sandro Pertini Hospital, Rome, Italy; ${ }^{20}$ Cardiology Department, Ramos Mejia Hospital, Buenos Aires, Argentina; ${ }^{21}$ Cardiodiagnosticos, Investigaciones Medicas, Buenos Aires, Argentina; ${ }^{22}$ Cardiology Division, Hospital San José, Criciuma, Brasil; ${ }^{23}$ Hospital Sao Vicente de Paulo e Hospital de Cidade, Passo Fundo, Brasil; ${ }^{24}$ Cardiology Institute of Rio Grande do Sul, Porto Alegre, Brasil; ${ }^{25}$ Hospital de Clinicas de Porto Alegre - Universidade Federal do Rio Grande do Sul, Porto Alegre, Brasil; ${ }^{26}$ Hospital San Vicente de Paul, Heredia, Costa Rica; ${ }^{27}$ Acibadem City Clinic Cardiovascular Center, University Hospital, Sofia, Bulgaria; ${ }^{28}$ Institute of Family Medicine, University of Szeged, and Department of Internal Medicine, Elisabeth Hospital, Hodmezovasarhely, Hungary; ${ }^{29}$ Centre of Cardiology and Angiology, Vilnius University Hospital Santaros Klinikos, Faculty of Medicine, Vilnius University, State Research Institute for Innovative Medicine, Vilnius, Lithuania; ${ }^{30}$ Chair of Cardiology, Bieganski Hospital, Medical University, Lodz Poland; ${ }^{31}$ Cardiology Clinic, Clinical Center of Serbia, Medical School, University of Belgrade, Serbia; ${ }^{32}$ Clinical Hospital Zvezdara Belgrade, Serbia; ${ }^{33}$ Department of Cardiology, Clinical Hospital Center Zemun, Faculty of Medicine, University of Belgrade, Serbia; ${ }^{34}$ Cardiology Department, University Clinic, Saint Petersburg, Russian Federation; ${ }^{35}$ Pediatric Cardiology Department, Brompton Hospital, London, UK, Division of Cardiology; ${ }_{i}^{36}$ Washington University School of Medicine, Barnes-Jewish Hospital, St. Louis, Missouri, USA; ${ }^{37}$ Hospital de Clinicas UFPR, Medicine Department, Federal University of Paranà, Curitiba, Brasil; ${ }^{38}$ Department of Cardiology, IRCCS Policlinico San Donato Clinic, Milan, Italy; ${ }^{39}$ Cardiology Research Institute, Tomsk National Tomsk National Research Medical Center of Russian Academy of Sciences; ${ }^{40}$ Cardiothoracic Department, University of Pisa, Italy; ${ }^{41}$ Heart Center, Hospital da Cruz Vermelha, Lisbon, and Medical School of University of Algarve, Faro, Portugal; ${ }^{42}$ Cardiology Department, Hospital Garcia de Orta, Almada, Portugal; ${ }^{43}$ Department of Cardiology, School of Health Sciences, Medical University of Silesia, Katowice, Poland; ${ }^{44}$ Department of Cardiology, Medical University of Białystok, Poland; ${ }^{45}$ Department of Cardiology, Wroclaw Medical University, Wroclaw, Poland; ${ }^{46}$ Department of Cardiology, Provincial Hospital, Szczecin, Poland; ${ }^{47}$ Hamad Medical Corporation, Heart Hospital, Doha, Qatar; ${ }^{48}$ Instituto Nacional de Cardiologia Ignacio Chavez, Mexico City, Mexico; ${ }^{49}$ Institute for Cardiovascular Diseases Dedinje, Belgrade, Serbia; ${ }^{50}$ Cardiology Division Ospedale Casilino, Roma Italy; ${ }^{51}$ Cardiology Department, County Hospital Sibiu, Invasive and Non-Invasive Center for Cardiac and Vascular Pathology in Adults - CVASIC Sibiu, Faculty of Medicine Sibiu, Romania; ${ }^{52}$ Cardiology Division Ospedale Civico Di Cristina Benfratelli Palermo; ${ }^{53}$ Cardiology Division, University Hospital, Palermo, Italy;

${ }^{54}$ University Clinical Center, Banja Luka, Republic of Srpska, Bosnia and Herzegovina; ${ }^{55}$ Department of Biostatistics, University of Padua, Padua, Italy.

Funding

Institutional funding from CNR Institute of Clinical Physiology. 


\section{Availability of data and materials}

Data sharing not applicable to this article as no data-sets were generated or analyzed during the current study.

See the stress echo 2020 website at: http://se2020.altervista.org/index.php/en/. (user name: reviewer; temporary password: N4ppGVgu70).

See the quality control content and modalities at: https://stressecho2020.mo odlecloud.com/login/index.php. (user name: reviewer; temporary password: N4ppGVgu70).

\section{Authors' contributions}

EP is the study chairman, designed the protocol, organized the content of webbased training and drafted the manuscript; QC is the principal investigator of SE2020, helped to organize the structure of training, contributed to developing the web-based training, critically revised the manuscript for an intellectually important contribution and approved the submitted version; MCS is the project leader of B-lines subproject in SE2020; MdN is the computer scientist who developed the website (SE 2020) and the web-based training material; MP is the computer scientist who organized and governed the quality control access, results, and data analysis; all other authors contributed to study design, undertook the quality control up to certification, are active members of SE 2020 consortium and critically revised the manuscript for an intellectually important contribution and approved the submitted version. RC and PC also coordinated the involvement of SIECVI (Società Italiana di Ecocardiografia e Cardiovascular Imaging). CC is responsible for data quality control and reader's certification.

\section{Ethics approval and consent to participate}

The study protocol was reviewed and approved by the institutional ethics committee as a part of the SE 2020 study (1487-CE Lazio-1, July 20, 2016).

\section{Consent for publication}

All the authors have read and approved the manuscript and accorded the consent for pubblication.

\section{Competing interests}

The authors declare that they have no competing interest.

\section{Publisher's Note}

Springer Nature remains neutral with regard to jurisdictional claims in published maps and institutional affiliations.

\section{Author details}

'CNR, Institute of Clinical Physiology, Biomedicine Department, Pisa, Italy. ${ }^{2}$ Cardiology Division, Fatebenefratelli Hospital, Benevento, Italy. ${ }^{3}$ Cardiology Department and Echocardiography Lab, University Hospital "San Giovanni di Dio e Ruggi d'Aragona", Salerno, Italy. ${ }^{4}$ Cardiology Hospital, Policlinico of Bari, Bari, Italy. ${ }^{5}$ Cardiology Department, San Carlo Hospital, Potenza, Italy. ${ }^{6}$ Cardiology Department, San Luca Hospital, Lucca, Italy. ${ }^{7}$ Cardiology Department, Echocardiography Lab, Monaldi Hospital, Second University of Naples, Naples, Italy. ${ }^{8}$ Casa di Cura Figlie di San Camillo, Cremona, Italy. ${ }^{9}$ Cardiology Department, Parma University Hospital, Parma, Italy.

${ }^{10}$ Department of Advanced Biomedical Sciences, Federico II University Hospital, Naples, Italy. ${ }^{11}$ Cardiology Department, Modena University Hospital, Modena, Italy. ${ }^{12}$ Cardio-Thorax-Vascular Department, Echocardiography lab, Policlinico Vittorio Emanuele, University of Catania, Catania, Italy. ${ }^{13}$ Cardiology Department, Careggi Hospital, Florence, Italy. ${ }^{14}$ Cardiology Division, Pontedera-Volterra Hospital, ASL Toscana 3 Nord-Ovest, Florence, Italy. ${ }^{15}$ Cardiology Department, San Camillo-Forlanini Hospital, Rome, Italy.

${ }^{16}$ Cardiology Department, Ospedale dell'Angelo Mestre-Venice, Venice, Italy. ${ }^{17}$ Cardiology Department, Nottola Hospital, Siena, Italy. ${ }^{18}$ Cardiology Department, Ospedale santa Maria Incoronata dell'Olmo, cava de' Tirreni, Salerno, Italy. ${ }^{19}$ Department of Cardiology, Sandro Pertini Hospital, Rome, Italy. ${ }^{20}$ Cardiology Department, Ramos Mejia Hospital, Buenos Aires, Argentina. ${ }^{21}$ Cardiodiagnosticos, Investigaciones Medicas, Buenos Aires, Argentina. ${ }^{22}$ Cardiology Division, Hospital San José, Criciuma, Brasília, Brazil. ${ }^{23}$ Hospital Sao Vicente de Paulo e Hospital de Cidade, Passo Fundo, Brazil. ${ }^{24}$ Cardiology Institute of Rio Grande do Sul, Porto Alegre, Brazil. ${ }^{25}$ Hospital de Clinicas de Porto Alegre - Universidade Federal do Rio Grande do Sul, Porto Alegre, Brazil. ${ }^{26}$ Hospital San Vicente de Paul, Heredia, Costa Rica. ${ }^{27}$ Acibadem City Clinic Cardiovascular Center, University Hospital, Sofia, Bulgaria.

${ }^{28}$ Institute of Family Medicine, University of Szeged, Szeged, Hungary.

${ }^{29} \mathrm{Centre}$ of Cardiology and Angiology, Vilnius University Hospital Santaros
Klinikos, Faculty of Medicine, Vilnius University, State Research Institute for Innovative Medicine, Vilnius, Lithuania. ${ }^{30}$ Chair of Cardiology, Bieganski Hospital, Medical University, Lodz, Poland. ${ }^{31}$ Cardiology Clinic, Clinical Center of Serbia, Medical School, University of Belgrade, Belgrade, Serbia. ${ }^{32}$ Clinical Hospital Zvezdara Belgrade, Belgrade, Serbia. ${ }^{33}$ Department of Cardiology, Clinical Hospital Center Zemun, Faculty of Medicine, University of Belgrade, Belgrade, Serbia. ${ }^{34}$ Cardiology Department, University Hospital, Saint Petersburg, Russian Federation. ${ }^{35}$ Pediatric Cardiology Department, Brompton Hospital, London, UK. ${ }^{36}$ Washington University School of Medicine, Barnes-Jewish Hospital, St. Louis, MO, USA. ${ }^{37}$ Hospital de Clinicas UFPR, Medicine Department, Federal University of Paranà, Curitiba, Brazil. ${ }^{38}$ Department of Cardiology, IRCCS Policlinico San Donato Clinic, Milan, Italy. ${ }^{39}$ Cardiology Research Institute, Tomsk National Research Medical Center of Russian Academy of Sciences, Tomsk, Russia. ${ }^{40}$ Cardiothoracic Department, University of Pisa, Pisa, Italy. ${ }^{41}$ Heart Center, Hospital da Cruz Vermelha, Lisbon and Medical School of University of Algarve, Faro, Portugal. ${ }^{42}$ Cardiology Department, Hospital Garcia de Orta, Almada, Portugal.

${ }^{43}$ Department of Cardiology, School of Health Sciences, Medical University of Silesia, Katowice, Poland. ${ }^{44}$ Department of Cardiology, Medical University of Białystok, Białystok, Poland. ${ }^{45}$ Department of Cardiology, Wroclaw Medical University, Wroclaw, Poland. ${ }^{46}$ Department of Cardiology, Provincial Hospital, Szczecin, Poland. ${ }^{47}$ Hamad Medical Corporation, Heart Hospital, Doha, Qatar. ${ }^{48}$ Instituto Nacional de Cardiologia Ignacio Chavez, Mexico City, Mexico. ${ }^{49}$ Institute for Cardiovascular Diseases, Dedinje, Belgrade, Italy. ${ }^{50} \mathrm{Cardiology}$ Division Ospedale Casilino, Rome, Italy. ${ }^{51}$ Cardiology Department, County Hospital Sibiu, Invasive and Non-Invasive Center for Cardiac and Vascular Pathology in Adults - CVASIC Sibiu, Faculty of Medicine, Sibiu, Romania. ${ }^{52}$ Cardiology Division Ospedale Civico Di Cristina Benfratelli, Palermo, Italy.

${ }^{53}$ Cardiology Division, University Hospital, Palermo, Italy. ${ }^{54}$ University Clinical

Center, Banja Luka, Republic of Srpska, Bosnia and Herzegovina.

\section{Received: 8 March 2018 Accepted: 3 August 2018}

Published online: 25 September 2018

\section{References}

1. Pellikka PA, Nagueh SF, Elhendy AA, Kuehl CA, Sawada SG. American Society of Echocardiography recommendations for performance, interpretation, and application of stress echocardiography. J Am Soc Echocardiogr. 2007;20:1021-4.

2. Sicari R, Nihoyannopoulos P, Evangelista A, Kasprzak J, Lancellotti P, Poldermans D. European Association of Echocardiography Stress echocardiography expert consensus statement: European Association of Echocardiography (EAE) (a registered branch of the ESC). Eur J Echocardiogr. 2008;9:415-37.

3. Picano E, Ciampi Q, Citro R, et al. Stress echo 2020 : The international Stress Echo study in ischemic and non-ischemic heart disease Cardiov Ultras 2017 ; Jan 18 15 (1): 3. DOl: https://doi.org/10.1186/s12947-016-0092-1

4. Gottdiener JS, Bednarz J, Devereux R, Gardin J, Klein A, Manning WJ, et al. American Society of Echocardiography. American Society of Echocardiography recommendations for use of echocardiography in clinical trials. J Am Soc Echocardiogr. 2004;17:1086-119.

5. Galderisi M, Henein MY, D' hooge J, Sicari R, Badano LP, Zamorano JL, Roelandt J. Recommendations of the European Association of Echocardiography. How to use echo-Doppler in clinical trials: different modalities for different purposes. Eur J Echocardiogr. 2011;12:339-53.

6. Feinstein AR. Diagnostic and spectral markers. Philadelphia: Clinical epidemiology. Saunders; 1985. p. 597-631.

7. Picano E, Landi P, Bolognese L, Chiarandà G, Chiarella F, Seveso G, et al. Prognostic value of dipyridamole echocardiography early after uncomplicated myocardial infarction: a large-scale, multicenter trial. The EPIC study group. Am J Med. 1993;95:608-18.

8. Picano E, Mathias W Jr, Pingitore A, Bigi R, Previtali M. Safety and tolerability of dobutamine-atropine stress echocardiography: a prospective, multicentre study. Echo Dobutamine international cooperative study group. Lancet. 1994;344:1190-2.

9. Ciampi Q, Picano E, Paterni M, Daros CB, Simova I, de Castro e Silva Pretto JL, D'Andrea A, Scali MC, Gaibazzi N, Severino S, Djordjevic-Dikic A, Kasprzak J, Zagatina A, Varga A, Lowenstein J, Merlo P, Amor M, Celeutkiene J, Perez JE, Di Salvo G, Galderisi M, Mori F, Costantino MF, Massa L, Dekleva M, Chavez D Q, Trambaiolo P, Citro R, Colonna P, Rigo F, Torres MAR, Monte I, Stankovic I, Neskovic A, Cortigiani L, Re F, Dodi C, D'Andrea A, Villari B, Arystan A, De Nes M, Carpeggiani C, on behalf of Stress Echo 2020. Quality 
control of regional wall motion analysis in stress Echo 2020. Int J Cardiol 2017:249479- 485

10. Picano E, Frassi F, Agricola E, Gligorova S, Gargani L, Mottola G. Ultrasound lung comets: a clinically useful sign of extravascular lung water. J Am Soc Echocardiogr. 2006;19:356-63.

11. Picano E, Pellikka PA. Ultrasound of extravascular lung water: a new standard for pulmonary congestion. Eur Heart J. 2016;37:2097-104.

12. Agricola E, Picano E, Oppizzi M, Pisani M, Zangrillo A, Margonato A. Assessment of stress-induced pulmonary interstitial edema by chest ultrasound during exercise echocardiography and its correlation with left ventricular function. J Am Soc Echocardiogr. 2006;19:457-63.

13. Scali MC, Cortigiani L, Simionuc A, Gregori D, Marzilli M, Picano E. The added value of exercise-echocardiography in heart failure patients: assessing dynamic changes in extravascular lung water. Eur J Heart Failure. 2017;19:1468-78

14. Simonovic D, Coiro S, Carluccio E, Girerd N, Deljanic-llic M, Ambrosio G. Exercise elicits dynamic changes in extravascular lung water and hemodynamic congestion in heart failure patients with preserved ejection fraction. Research letter. Eur J Heart Fail. 2018;21. https://doi.org/10.1002/ ejhf.1228. [Epub ahead of print]

15. Lancellotti P, Pellikka PA, Budts W, Chaudhry FA, Donal E, Dulgheru R, Edvardsen T, Garbi M, Ha JW, Kane GC, Kreeger J, Mertens L, Pibarot P, Picano E, Ryan T, Tsutsui JM, Varga A. The clinical use of stress echocardiography in non-ischaemic heart disease: recommendations from the European Association of Cardiovascular Imaging and the American Society of Echocardiography. Eur Heart J Cardiovasc Imaging. 2016;17:1191-229.

16. Scali MC, Zagatina A, Simova I, Zhuravskaya N, Ciampi Q, Paterni M, Marzilli M, Carpeggiani C. Picano E. B-lines with Lung Ultrasound: the optimal scan technique at rest and during stress Ultrasound Med Biol. 2017;43:2558-66.

17. Picano E, Scali MC, Ciampi Q, Lichtenstein D. Lung ultrasound for the cardiologist. JACC imaging. 2018;12:381-90.

18. Mayo PH, Beaulieu Y, Doelken P, et al. American College of Chest Physicians/La Société de Réanimation de Langue Française statement on competence in critical care ultrasonography. Chest. 2009;135:1050-60.

19. Beaulieu Y, Laprise R, Drolet P, Thivierge RL, Serri K, Albert M, Lamontagne A, Belliveau M, Denault AY, Patenaude JV. Bedside ultrasound training using web-based e-learning and simulation early in the curriculum of residents. Critical Ultrasound Journal. 2015;7:1

20. Sun Lim J, Lee S, Ho Do H, Ho Oh K. Can Limited Education of Lung Ultrasound Be Conducted to Medical Students Properly? A Pilot Study BioMed Research International Volume 2017, Article ID 8147075, 6 pages doi https://doi.org/10.1155/2017/8147075

21. Gargani L, Sicari R, Raciti M, Serasini L, Passera M, Torino C, Letachowicz K, Ekart R, Fliser D, Covic A, Balafa O, Stavroulopoulos A, Massy ZA, Fiaccadori E, Caiazza A, Bachelet T, Slotki I, Shavit L, Martinez-Castelao A, Coudert-Krier MJ, Rossignol P, Kraemer TD, Hannedouche T, Panichi V, Wiecek A, Pontoriero G, Sarafidis P, Klinger M, Hojs R, Seiler-Mußler S, Lizzi F, Onofriescu M, Zarzoulas F, Tripepi R, Mallamaci F, Tripepi G, Picano E, London GM, Zoccali C. Efficacy of a remote web-based lung ultrasound training for nephrologists and cardiologists: a LUST trial sub-project. Nephrology Dialysis Transplantation. 2016:31:1982-8.

22. Lindseth F, Hallan ML, Tonnessen MS, Smistad E, Vapenstad C. MIIP: a webbased platform for medical image interpretation training and evaluation focusing on ultrasound. Proceedings Volume 10138, Medical Imaging 2017: Imaging Informatics for healthcare Research and Applications; 10138W; doi: 10.117/12.2254158

23. Jambrik Z, Monti S, Coppola V, Agricola E, Mottola G, Picano E. Usefulness of ultrasound lung comets as a nonradiologic sign of extravascular lung water. Am J Cardiol. 2004;93:1265-70.

24. Volpicelli G, Elbarbary M, Blaivas M, et al. International Liaison Committee on Lung Ultrasound for International Consensus Conference on Lung Ultrasound International evidence-based recommendations for point-of-care lung ultrasound. Intensive Care Med. 2012;38:577-91

25. Platz E, Pivetta E, Merz AA, Peck J, Rivero J, Cheng S. Impact of device selection and clip duration on lung ultrasound assessment in patients with heart failure. Am J Emerg Med. 2015:33:1552-6.

26. Ohman J, Harjola VP, Karjalainen P, Lassus J. Assessment of early treatment response by rapid cardiothoracic ultrasound in acute heart failure: cardiac filling pressures, pulmonary congestion and mortality. Eur Heart J Acute Cardiovasc Care. 2018;7:311-20.
27. Picano E, Scali MC. The lung water cascade in heart failure. Echocardiography. 2017:34:1503-7.

28. Picano E, Scali MC. Stress echo, carotid arteries and more: its versatility for our imaging times. Editorial comment JACC img. 2017; https://doi.org/10. 1016/j.jcmg.2017.01.023

29. Picano E, Pellikka PA. Stress echo applications beyond coronary artery disease. Eur Heart J. 2014:35:1033-40

30. Messerli FH. Meta-analysis and bouillabaisse. Ann Intern Med. 1996;125:519.
Ready to submit your research? Choose BMC and benefit from:

- fast, convenient online submission

- thorough peer review by experienced researchers in your field

- rapid publication on acceptance

- support for research data, including large and complex data types

- gold Open Access which fosters wider collaboration and increased citations

- maximum visibility for your research: over $100 \mathrm{M}$ website views per year

At $\mathrm{BMC}$, research is always in progress.

Learn more biomedcentral.com/submissions 